\title{
RESEARCH
}

Open Access

\section{Developmental changes in attention to social information from childhood to adolescence in autism spectrum disorders: a comparative study}

Toru Fujioka ${ }^{1,2,3+}$, Kenji J. Tsuchiyaa ${ }^{3,4+}$, Manabu Saito ${ }^{5}$, Yoshiyuki Hirano ${ }^{3,6}$, Muneaki Matsuo ${ }^{7}$, Mitsuru Kikuchi ${ }^{3,8}$, Yoshihiro Maegaki ${ }^{9}$, Damee Choi ${ }^{3,4}$, Sumi Kato ${ }^{5}$, Tokiko Yoshida ${ }^{6}$, Yuko Yoshimura ${ }^{10}$, Sawako Ooba ${ }^{11}$, Yoshifumi Mizuno ${ }^{12}$, Shinichiro Takiguchi, ${ }^{3,12}$, Hideo Matsuzaki ${ }^{2,3}$, Akemi Tomoda, ${ }^{2,}$, Katsuyuki Shudo ${ }^{13}$, Masaru Ninomiya ${ }^{13}$, Taiichi Katayama ${ }^{3}$ and Hirotaka Kosaka ${ }^{2,3,14^{*}}$

\begin{abstract}
Background: Elucidating developmental changes in the symptoms of autism spectrum disorder (ASD) is important to support individuals with ASD. However, no report has clarified the developmental changes in attention to social information for a broad age range. The aim of this study was to investigate the developmental changes in attention to social information from early childhood to adolescence in individuals with ASD and typically developed (TD) children.

Methods: We recruited children with ASD $(n=83)$ and TD participants $(n=307)$ between 2 and 18 years of age. Using the all-in-one-eye-tracking system, Gazefinder, we measured the percentage fixation time allocated to areas of interest (Aols) depicted in movies (the eyes and mouth in movies of a human face with/without mouth motion, upright and inverted biological motion in movies showing these stimuli simultaneously, people and geometry in preference paradigm movies showing these stimuli simultaneously, and objects with/without finger-pointing in a movie showing a woman pointing toward an object). We conducted a three-way analysis of variance, 2 (diagnosis: ASD and TD) by 2 (sex: male and female) by 3 (age group: $0-5,6-11$, and $12-18$ years) and locally weighted the scatterplot smoothing (LOESS) regression curve on each Aol.
\end{abstract}

Results: In the face stimuli, the percentage fixation time to the eye region for the TD group increased with age, whereas the one for the ASD group did not. In the ASD group, the LOESS curves of the gaze ratios at the eye region increased up to approximately 10 years of age and thereafter tended to decrease. For the percentage fixation time to the people region in the preference paradigm, the ASD group gazed more briefly at people than did the TD group. (Continued on next page)

\footnotetext{
*Correspondence: hirotaka@u-fukui.ac.jp

${ }^{\dagger}$ Toru Fujioka and Kenji J. Tsuchiya contributed equally to this work.

${ }^{2}$ Research Center for Child Mental Development, University of Fukui, Eiheiji,

Fukui, Japan

${ }^{3}$ Department of Child Development, United Graduate School of Child

Development, Osaka University, Kanazawa University, Hamamatsu University

School of Medicine, Chiba University, and University of Fukui, Suita, Osaka,

Japan

Full list of author information is available at the end of the article
}

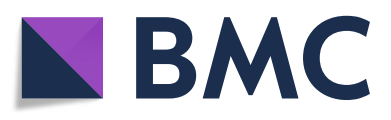

(- The Author(s). 2020 Open Access This article is licensed under a Creative Commons Attribution 4.0 International License, which permits use, sharing, adaptation, distribution and reproduction in any medium or format, as long as you give appropriate credit to the original author(s) and the source, provide a link to the Creative Commons licence, and indicate if changes were made. The images or other third party material in this article are included in the article's Creative Commons licence, unless indicated otherwise in a credit line to the material. If material is not included in the article's Creative Commons licence and your intended use is not permitted by statutory regulation or exceeds the permitted use, you will need to obtain permission directly from the copyright holder. To view a copy of this licence, visit http://creativecommons.org/licenses/by/4.0/ The Creative Commons Public Domain Dedication waiver (http://creativecommons.org/publicdomain/zero/1.0/) applies to the data made available in this article, unless otherwise stated in a credit line to the data. 


\begin{abstract}
(Continued from previous page)
Limitations: It is possible that due to the cross-sectional design, the degree of severity and of social interest might have differed according to the subjects' age.

Conclusions: There may be qualitative differences in abnormal eye contact in ASD between individuals in early childhood and those older than 10 years.
\end{abstract}

Keywords: Autism spectrum disorder, Social information, Eye-tracking, Developmental change

\section{Background}

Autism spectrum disorder (ASD) is a neurodevelopmental disorder characterized by a "deficit of social communication and social interaction" and "restricted, repetitive patterns of behavior, interests, or activities," comprising several specific symptoms [1]. The core features of ASD persist throughout life. However, it is considered that the clinical presentation of ASD may change during development [2,3]. It would be beneficial for diagnosis, treatment, and support to clarify changes in the clinical picture of ASD associated with the developmental stages.

Most, but not all, previous studies have reported that individuals with ASD gaze less at social information regardless of age. For example, the face, especially the eye and mouth regions, conveys salient social information, and it is believed that people prefer to pay attention to these areas from infancy [4]. When shown videos and photographs in which people appear, children with ASD [5-12] and adults with ASD [13] are both reported to gaze at the human faces for shorter periods of time than do typically developed (TD) individuals. Studies with various types of stimuli have reported that, of the parts of the face, the region around the eyes is particularly looked closely at more briefly, by both children with ASD [9, 12, 14-20] and adults with ASD [11, 18, 2125 . When the TD and ASD groups were simultaneously shown movies of people and geometric shapes, i.e., the preference paradigm, which was created under the hypothesis that individuals with ASD prefer to pay attention to highly repetitive images than to social images [26], the ASD group observed people for shorter periods of time than did the TD group. This gaze behavior has been reported in both children with ASD [26-30] and adults with ASD [22]. Moreover, when TD children and children with ASD were shown movies of biological motion, in which multiple points moved in concert and created movements that resemble those of living beings, children with ASD were reported to gaze at biological motion for shorter periods than did the TD children [31-33]. It is considered that the preference for biological motion is a fundamental mechanism facilitating adaptive interaction with other living beings [31] and that the lesser attention to biological motion in the ASD groups appears to be more specific to the processing of biological motion than to a purely perceptual problem
[34]. In a video of joint attention that used fingerpointing movements, recognized as an equally attractive social stimulus for humans as that described above [4], children with ASD reportedly reacted less accurately to finger-pointing than did the TD children [35] or showed a lower ratio of gazing at an object to which a finger had been pointed [36]. However, when presented only with people's faces on a monitor in a passive view condition, children in an ASD group showed comparable attention to the eye region with those in a TD group [5, 37], while post-pubescent participants in an ASD group showed less attention to the eye region than did post-pubescent participants in a TD group [14, 22]. Group differences between ASD and TD were more likely to occur with dynamic stimuli containing several individuals than with dynamic stimuli containing a single individual [16]. Moreover, group differences between ASD and TD in attention to social information did not occur on biological motion in adults [22], on the preference paradigm when the stimuli contained only one individual in children [27], and on cartoon-like images with a human figure in school-aged children [38]. Thus, although individuals with ASD tend not to pay attention to social information, this appears to ultimately depend on the quality of the stimulus and the participant's age.

This gazing at social information reportedly changes during development, and the developmental trajectories between ASD and TD groups appear to differ. For example, when a scene from a TV program or a photograph of a person's face was shown to both TD adults/ children and adults/children with ASD, TD children tended to gaze more briefly at the eye region than did TD adults; however, it was reported that no significant differences between children and adults with ASD were observed in the gaze ratio at the eyes and mouth regions $[11,18]$. In addition, a study presented a video of a face, the preference paradigm, biological motion, and fingerpointing to TD children aged $4.0 \pm 1.9$ years and children with ASD aged $4.8 \pm 1.1$ years [37]. In the preference paradigm, the gaze ratio at the people region decreased and that of the geometric shapes increased with age in both the ASD and TD groups. In the TD group, the gaze ratio at the region around the mouth in a video of a face and the ratio of gazing at the nonobject being pointed at in the movie of finger-pointing 
increased with age. In addition, the ratio of gazing at the object being pointed at in the movie of finger-pointing decreased with age in the TD group. Conversely, the participants in the ASD group did not show other significant correlations. Although a weakness of that study was that the sample size was small, the results showed that participants with ASD did not show the developmental changes in attention to social information that TD participants showed in childhood [37]. To summarize, depending on the type of stimulus, participants in the ASD group appeared not to show the maturation of the fixation pattern for faces that occurs from childhood to adulthood in TD individuals.

However, no study targeting either TD individuals or individuals with ASD has thus far identified the developmental changes in gazing at social information targeting subjects covering a broad age range after childhood. In addition, there is a discrepancy, especially in TD, in that although gazing at areas carrying strong social information decreases or does not change along with increasing age in childhood, adults often gaze longer at such information than do children. Identifying the developmental changes in gazing at social information, targeting a broad range of age groups after childhood, will help resolve this contradiction. It is also assumed that developmental changes in gazing at social information depend on the quality of the stimulation; hence, identifying the quality of stimulation that the subjects react to sensitively would offer suggestions for elucidating the mechanism by which social difficulties occur in children and adults with ASD. In this study, therefore, we aimed at identifying the developmental changes in gazing at social information in individuals with ASD and TD, from childhood to adolescence. We used Gazefinder (JVC KENWOOD Corporation, Kanagawa, Japan), an eyetracking device that can measure gazing at social information with multiple stimuli of different qualities.

\section{Methods \\ Participants \\ The ASD group}

A total of 108 children between 3 and 17 years old (18 boys and 8 girls in the $0-5$-year group, 37 boys and 10 girls in the 6-11-year group, and 25 boys and 10 girls in the 12-18-year group) with ASD were recruited from the University of Fukui Hospital, Hamamatsu University Hospital, Hirosaki University Hospital, Chiba University Hospital, Kanazawa University Hospital, Tottori University, Saga University Hospital, and Osaka University Hospital, Japan. All participants were of Japanese ethnicity. The participants were diagnosed with ASD or any other psychopathological condition by a certified psychiatrist of the Japanese Board of Psychiatry or boardcertified pediatrician of the Japan Pediatric Society, based on the criteria of the Diagnostic and Statistical Manual of Mental Disorders, fifth edition [1]. Although we permitted attention deficit hyperactivity disorder as a comorbid disorder, we excluded participants that met the diagnostic criteria for any other psychopathological condition. The IQ of all participants with ASD was assessed with the Wechsler Intelligence Scale for Children-Fourth Edition (WISC-IV), or the TanakaBinet Test (Japanese version of the Stanford-Binet Test), or the developmental quotient (DQ) of the Kyoto Scale of Psychological Development. All participants had an IQ/DQ of 70 or higher.

\section{TD group}

We also recruited 374 TD participants between 2 and 18 years old ( 81 boys and 78 girls in the $0-5$-year group, 80 boys and 89 girls in the $6-11$-year group, and 20 boys and 26 girls in the 12-18-year group) from the local community. All participants were of Japanese ethnicity. On the face sheet of the questionnaire, we confirmed that for the TD participants, there was no indication of disorder at the medical checkups for 1.5-year-old and 3year-old children conducted by pediatricians and public health nurses in Japan, and that they had not been diagnosed with mental disorders and had no active diseases requiring continuous hospital visits.

\section{Stimuli}

We utilized Gazefinder, an all-in-one eye-tracking system where hardware and stimulating videos are grouped together to evaluate the percentages of fixation time allocated to specific objects (see below) on a video monitor. Participant eye positions were measured using infrared light sources and cameras located below a 19-inch thin-film transistor $(1280 \times 1024$ pixels $)$. Using corneal reflection techniques, eye position was recorded as (X, Y) coordinates at a frequency of $50 \mathrm{~Hz}$ (i.e., 3000 data collections/min). The calibration of eye position recordings was performed using a five-point method. It is recommended to retain the distance between the face and the monitor at approximately $70 \mathrm{~cm}$.

After calibration of the eye position with a five-point method, Gazefinder presented five types of movies; (A) human faces without mouth motion, (B) human faces with mouth motion, $(C)$ biological motion of a human, (D) the preference paradigm, and (E) finger-pointing. (A) Human faces without mouth motion included movies of a still face ( $4 \mathrm{~s}$ ), of the eyes blinking (an actress repeatedly opens and closes her eyes for $5 \mathrm{~s}$ ), and of a still face (an actress with a still face appears for $5 \mathrm{~s}$, and this movie was presented after the mouth moving face movie described below). (B) Human faces with mouth motion included movies of a mouth moving face (an actress repeatedly opens and closes her mouth for $5 \mathrm{~s}$ ) and 
of a talking face $(7 \mathrm{~s})$. In the talking face movie, the actress says, "Konnichiwa" ("Hello"), "Onamaewa?" ("What is your name?"), and "Issyoniasobouyo" ("Let's play together"). Face stimuli are considered representative of social stimuli [4], and humans appear to naturally pay attention to the face, especially to the eye area [39]. The difference between "(A) human faces without mouth motion" and "(B) human faces with mouth motion" is that the moving mouth interferes with directing attention to the eye region. Moreover, previous studies have reported that attention to the mouth region increases when individuals are shown movies of talking faces or of faces with the mouth in motion [22, 39]. (C) Biological motion movies presented upright and inverted biological motion simultaneously for $11 \mathrm{~s}$. The movie was accompanied by the song "Under the Big Chestnut Tree" to which an upright human danced. The biological motion movie measures the degree of attention to social information under the hypothesis that humans show an innate preference for a biological motion to facilitate adaptive interaction with other living beings [31]. In addition, a brain imaging study with adults suggested that the activated brain areas processing inverted biological motion differed from those processing upright biological motion [40]. (D) The preference paradigm movies simultaneously showed people and geometric shapes at the same size $(20 \mathrm{~s})$ and geometric shapes in small-frame images in a small window embedded in movies of people $(16 \mathrm{~s})$. The preference paradigm movie measures the degree of attention to social information under the hypothesis that individuals with ASD prefer to pay attention to highly repetitive images rather than social images [26]. (E) Finger-pointing movies presented objects with or without finger-pointing (8s). Fingerpointing, also known as joint attention, concerns a shared attention state between two individuals focused on an object/event of interest [41] and is categorized as representative of social stimuli [4], as described above. Therefore, it is suggested that the degree of attention to social information can be determined by measuring the response to these types of stimuli. Each stimulus was presented once, and the presentation order of these stimuli was randomly predetermined; all participants saw the stimuli in the same order. A music box sound was played while presenting the stimuli except for $(C)$ biological motion. Figure 1 presents samples of the stimuli.

Percentage fixation times allocated to areas of interest (AoIs) on the video monitor were automatically calculated (time allocated to a particular area/duration of stimulus presentation). The stimulus movies were loaded on Gazefinder, and the AoIs of each stimulus were also set by default; therefore, there was no need for the experimenter to change the setting to derive the percentage fixation times allocated to the AoIs. The AoIs in each stimulus are presented in Fig. 1.

\section{Procedures}

The study protocol was approved by the ethics committee of each university and conformed to the tenets of the Declaration of Helsinki (as revised in 2000). After a complete explanation of the study, all participants or their parents/ legal guardians provided written informed consent. Participants completed the Gazefinder test in a quiet room in each facility. For the ASD group, IQ/DQ evaluation was performed if it had not taken place within the past 2 years. The parents/legal guardians of the participants completed the Social Responsiveness Scale Second Edition (SRS-2), which is a questionnaire used to determine the severity of social deficits [42]. The SRS-2 consists of 65 items, and total SRS scores range from 0 to 195, with higher total scores indicating more severe social deficits. There are SRS-2 forms for preschool children (2.5-4.5 years), school-age children (4-18 years), and adults (ages 19 and up). We used the SRS-2 preschool form for participants aged 2 to 3 years and the SRS-2 school-age form for participants older than 4 years.

\section{Analysis of data}

Exclusion criteria

As a reference to the Japanese cutoff score of the SRS2 , the cutoff score for the SRS-2 preschool form was 48.5 (sensitivity, 0.83; specificity, 0.82) [43], and the cutoff scores for the SRS-2 school-age form were 53.5 for boys (sensitivity, 0.91; specificity, 0.48) and 52.5 for girls (sensitivity, 0.89; specificity, 0.41) [44]; we excluded participants with ASD who were below and TD participants who were above the cutoff point. Additionally, individuals were excluded when their available percentage of fixation time was $<70 \%$ (i.e., Gazefinder could not detect the eye position more than $30 \%$ of the stimulus presentation time).

\section{Statistical analysis}

All statistical analyses were conducted using IBM SPSS, version 24 (IBM Corp., Armonk, NY). At first, for age comparisons, we carried out two-way analysis of variance (ANOVA), 2 (diagnosis: ASD and TD) by 2 (sex: male and female), for each age group (age groups: 0-5year group, 6-11-year group, and 12-18-year group). We set .05 as the significance level. Second, we conducted a three-way ANOVA for the SRS-2 total score, 2 (diagnosis) by 2 (sex) by 3 (age group). We set .05 as the significance level. Third, we conducted a three-way ANOVA on each AoI, 2 (diagnosis) by 2 (sex) by 3 (age group), for clarifying the effects in gaze patterns of these factors. To avoid type 1 statistical errors, we applied Bonferroni corrections and set .05 divided by the total 


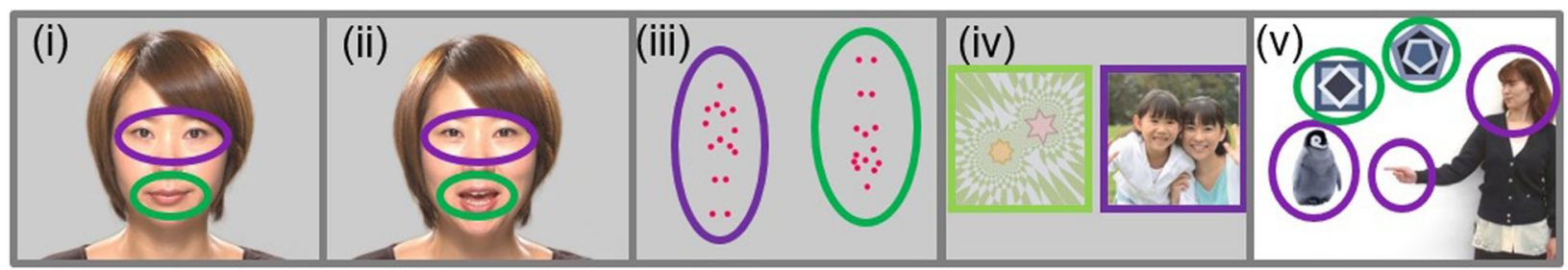

Fig. 1 Gazefinder movie samples and their areas of interest (Aols). (i) Screenshot of the human face without mouth motion; Aol-1 and Aol-2 include the eye and mouth regions, respectively; (ii) Screenshot of the human face with mouth motion; Aol-1 and Aol-2 include the eye and mouth regions, respectively; (iii) Screenshot of biological motion; Aol-1 and Aol-2 are the upright and inverted images, respectively; (iv) Screenshot of the preference paradigm; Aol-1 and Aol-2 are people and geometry, respectively; (v) Screenshot of finger pointing; Aol-1 and Aol-2 are social and geometry areas, respectively

number of AoIs as the significance level; thus, we set $p=$ $.005(.05 / 10)$ as the significance level. We also applied Bonferroni corrections for post hoc comparisons. However, we could only grasp rough developmental changes in gazing at social information using this method. In addition, as described above, developmental changes in gazing at social information were unknown. Therefore, we finally used locally weighted scatterplot smoothing (LOESS) $[45,46]$ to graphically evaluate the continuous effect of age on attention to social information. LOESS is a procedure for fitting a regression surface to data through multivariate smoothing [46]. We used the LOESS kernel Epanechnikov method and set the \% of points to fit to $50 \%$, which is the default value of LOESS analysis in IBM SPSS version 24. We used the kernel Epanechnikov method, which is one of the most popular, for pattern analysis [47]. Finally, with consideration to the analysis results described above, we carried out a correlation analysis between the SRS-2 scores and the percentage fixation time to AoIs and correlation analysis between the IQ/DQ scores and the percentage fixation time to AoIs. As IQ/DQ was only measured in the ASD group, the latter is only for the ASD group. We set $p=$ .005 as the significance level following the rationale used for the previous analyses.

\section{Results}

Applying the exclusion criteria described above, there were 83 participants in the ASD group (63 boys and 20 girls): 19 (13 boys and 6 girls) in the $0-5$-year group, 34 ( 28 boys and 6 girls) in the $6-11$-year group, and 30 (22 boys and 8 girls) in the 12-18-year group. In the TD group, the final participants were 307 (141 boys and 166 girls); the 0-5-year group included 120 (58 boys and 62 girls) participants, the 6-11-year group, 150 (69 boys and 81 girls) participants; and the 12-18-year group, 37 ( 14 boys and 23 girls) participants. The characteristics of the participants included in the analysis are summarized in Table 1.
For age, in the 6-11-year group, there was a significant main effect of age group $\left(F(1,180)=4.29, p=.040, \eta^{2}\right.$ $=0.023)$, with the participants in the ASD group being significantly older than those in the TD group. In the 12-18-year group, there was a significant main effect of $\operatorname{sex}\left(F(1,63)=7.29, p=.009, \eta^{2}=0.104\right)$, with female participants being significantly older than male participants. Other main effects and interactions were not significant. For SRS-2, there was a significant main effect of diagnosis $\left(F(1,378)=673.76, p<.001, \eta^{2}=0.641\right)$, and a diagnosis $\times$ age group interaction $(F(2,378)=8.35, p$ $<.001, \eta^{2}=0.042$ ). Post hoc comparisons applying Bonferroni corrections revealed that the SRS-2 total score in the $0-5$-year group was significantly higher than those in the other age groups in TD group and lower than those in the other age groups in ASD group.

\section{Face without mouth motion}

For the eye region, there was a significant main effect of age group $\left(F(2,378)=6.68, p=.001, \eta^{2}=0.034\right)$ and none of the other main effects and interactions were significant ( $\mathrm{Fs} \leq 5.20$, ps $\geq .023$ ). Post hoc comparisons, applying Bonferroni corrections, revealed that the percentage fixation times in the 12-18-year group were significantly higher than those in the other groups (ps < .001 ), and the percentage fixation times in the 6-11-year group were significantly higher than those in the $0-5$ year group $(p<.001)$. Based on the LOESS plot, the ASD group showed a sharp decline of attention to the eye region after around 10 years of age, while there was a constant rise of attention to the eye region after around 5 years of age in the TD group.

For the mouth region, the diagnosis $\times$ age group interaction was significant $\left(F(2,378)=5.80, p=.003, \eta^{2}=\right.$ 0.030 ), and the percentage fixation times in the 12-18year group were significantly lower than those in the 0 5 -year and $6-11$-year groups in the TD group $(p<.001)$. There were no significant differences among the age groups in the ASD group $(p>.10)$. The LOESS plot showed that there was a sharp increase of attention to 
Table 1 Characteristics of the participants

\begin{tabular}{|c|c|c|c|c|c|c|}
\hline & & $n$ & Age & IQ/DQ & SRS-2 & Comorbid ADHD \\
\hline \multicolumn{7}{|l|}{ ASD } \\
\hline \multirow[t]{3}{*}{ 0-5-year group } & Total & 19 & $4.7 \pm 0.9$ & $89.1 \pm 10.3$ & $72.8 \pm 17.3$ & 10 \\
\hline & Male & 14 & $4.6 \pm 1.0$ & $89.1 \pm 9.3$ & $70.0 \pm 19.0$ & 8 \\
\hline & Female & 5 & $5.0 \pm 0.5$ & $89.2 \pm 13.9$ & $80.6 \pm 8.7$ & 2 \\
\hline \multirow[t]{3}{*}{ 6-11-year group } & Total & 34 & $9.2 \pm 1.6$ & $94.6 \pm 14.6$ & $88.5 \pm 25.6$ & 17 \\
\hline & Male & 28 & $9.0 \pm 1.6$ & $95.0 \pm 15.1$ & $88.3 \pm 26.7$ & 15 \\
\hline & Female & 6 & $10.0 \pm 1.6$ & $92.7 \pm 13.0$ & $89.3 \pm 21.2$ & 2 \\
\hline \multirow[t]{3}{*}{ 12-18-year group } & Total & 30 & $14.5 \pm 1.6$ & $97.3 \pm 14.6$ & $88.3 \pm 24.7$ & 14 \\
\hline & Male & 22 & $14.4 \pm 1.7$ & $101.0 \pm 13.7$ & $88.7 \pm 23.1$ & 11 \\
\hline & Female & 8 & $14.9 \pm 1.4$ & $87.3 \pm 12.8$ & $87.3 \pm 30.5$ & 3 \\
\hline \multicolumn{7}{|l|}{ TD } \\
\hline \multirow[t]{3}{*}{ 0-5-year group } & Total & 120 & $4.6 \pm 1.0$ & - & $29.6 \pm 11.8$ & - \\
\hline & Male & 58 & $4.5 \pm 1.0$ & - & $31.3 \pm 10.7$ & - \\
\hline & Female & 62 & $4.7 \pm 1.0$ & - & $28.0 \pm 12.6$ & - \\
\hline \multirow[t]{3}{*}{ 6-11-year group } & Total & 150 & $8.7 \pm 1.7$ & - & $23.8 \pm 11.8$ & - \\
\hline & Male & 69 & $8.6 \pm 1.6$ & - & $24.4 \pm 11.8$ & - \\
\hline & Female & 81 & $8.8 \pm 1.7$ & - & $23.3 \pm 11.9$ & - \\
\hline \multirow[t]{3}{*}{ 12-18-year group } & Total & 37 & $14.2 \pm 1.5$ & - & $21.5 \pm 11.7$ & - \\
\hline & Male & 14 & $13.2 \pm 0.8$ & - & $19.7 \pm 10.3$ & - \\
\hline & Female & 23 & $14.8 \pm 1.6$ & - & $22.5 \pm 12.6$ & - \\
\hline
\end{tabular}

Table 2 Results of the ANOVA

\begin{tabular}{|c|c|c|c|c|c|c|c|c|c|c|c|c|c|c|}
\hline & \multicolumn{2}{|c|}{ Diagnosis } & \multicolumn{2}{|l|}{ Sex } & \multicolumn{2}{|c|}{ Age group } & \multicolumn{2}{|c|}{$\begin{array}{l}\text { Diagnosis } \times \\
\text { sex }\end{array}$} & \multicolumn{2}{|c|}{$\begin{array}{l}\text { Diagnosis } \times \text { age } \\
\text { group }\end{array}$} & \multicolumn{2}{|c|}{$\begin{array}{l}\text { Sex } \times \text { age } \\
\text { group }\end{array}$} & \multicolumn{2}{|c|}{$\begin{array}{l}\text { Diagnosis } \times \text { sex } \times \text { age } \\
\text { group }\end{array}$} \\
\hline & $F$ & $P$ & $F$ & $P$ & $F$ & $P$ & $F$ & $P$ & $F$ & $P$ & $F$ & $P$ & $F$ & $P$ \\
\hline \multicolumn{15}{|c|}{ Face without mouth motion } \\
\hline Eye & 5.20 & .023 & 0.20 & .652 & 6.68 & $.001 *$ & 2.30 & .130 & 4.36 & .013 & 1.46 & .233 & 4.41 & .013 \\
\hline Mouth & 1.33 & .249 & 1.41 & .235 & 1.26 & .286 & 0.11 & .745 & 5.80 & $.003^{*}$ & 0.47 & .628 & 2.05 & .131 \\
\hline \multicolumn{15}{|c|}{ Face with mouth motion } \\
\hline Eye & 4.79 & .029 & 0.00 & .985 & 8.09 & $<.001^{*}$ & 2.94 & .087 & 9.43 & $<.001^{*}$ & 0.60 & .549 & 8.83 & $<.001 *$ \\
\hline Mouth & 0.24 & .626 & 0.01 & .931 & 4.26 & .015 & 1.77 & .184 & 4.31 & .014 & 0.01 & .989 & 3.57 & .029 \\
\hline \multicolumn{15}{|c|}{ Biological motion } \\
\hline Upright & 0.25 & .616 & 0.11 & .746 & 1.51 & .221 & 0.24 & .622 & 0.62 & .539 & 0.34 & .715 & 0.90 & .406 \\
\hline Inverted & 7.51 & .006 & 0.00 & .999 & 1.45 & .237 & 0.08 & .774 & 1.06 & .347 & 0.28 & .757 & 0.98 & .377 \\
\hline \multicolumn{15}{|c|}{ The preference paradigm } \\
\hline People & 8.36 & $.004^{*}$ & 10.39 & $.001 *$ & 3.73 & .025 & 0.43 & .511 & 1.88 & .154 & 0.02 & .982 & 0.22 & .802 \\
\hline Geometry & 4.31 & .039 & 10.59 & $.001 *$ & 6.09 & $.003^{*}$ & 2.18 & .140 & 1.17 & .311 & 0.90 & .407 & 0.36 & .700 \\
\hline \multicolumn{15}{|c|}{ Finger-pointing } \\
\hline Social & 3.09 & .080 & 0.65 & .420 & 1.08 & .342 & 0.63 & .429 & 0.19 & .830 & 0.29 & .752 & 0.02 & .976 \\
\hline Geometry & 14.96 & $<.001^{*}$ & 0.00 & .957 & 0.66 & .517 & 2.06 & .152 & 0.32 & .725 & 1.04 & .355 & 1.36 & .258 \\
\hline
\end{tabular}


the mouth region in the ASD group after around 10 years of age, whereas there was a constant decline of attention to the mouth region in the TD group after around 5 years of age.

These results are shown in Table 2 and Fig. 2

\section{Face with mouth motion}

For the eye region, the analysis revealed a three-way interaction $\left(F(2,378)=8.83, p<.001, \eta^{2}=0.045\right)$. Post hoc comparisons showed that the male participants in the TD 12-18-year group gazed significantly longer at the eye region than did the TD male $0-5$-year group $(p$ $=.001)$, and the female participants in the TD 12-18year group gazed significantly longer at the eye region than did the participants in $0-5$ and 6-11-year groups (ps < .001). Conversely, in both ASD sex groups, there were no age-group differences (ps $>.050$ ). Furthermore, there were sex differences only in the 12-18-year group for both the ASD and TD groups, while the TD female group gazed significantly longer at the eye region than did the TD male group $(p=.006)$; the ASD female group gazed significantly less at the eye region than did the ASD male group $(p=.003)$. As a significant sex difference was found on the ANOVA, we present separate LOESS plots for each sex in Fig. 3. Especially remarkable in girls, the regression line in both ASD groups showed a sharp decline of attention to the eye region after around 10 years of age. Conversely, there was a constant rise of attention to the eye region in the TD group.

There were no main effects and interactions for the mouth region ( $\mathrm{Fs} \leq 4.31$, ps $\geq .014$ ). As is the case with the face without mouth motion, the LOESS plot in Fig. 3

\section{Face without mouth motion}

(a) Eye

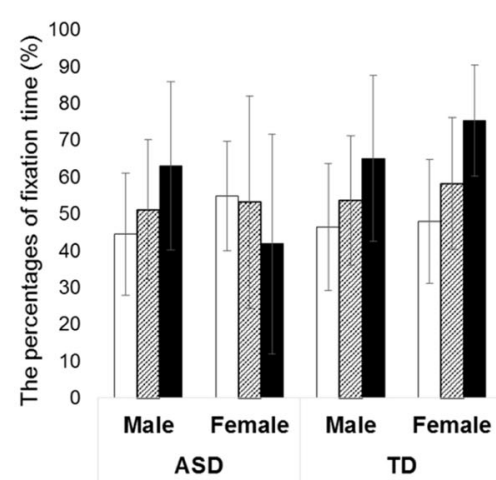

(c) ASD

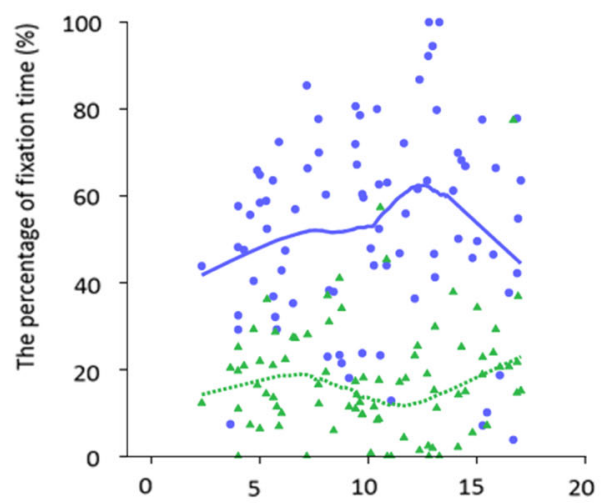

(b) Mouth

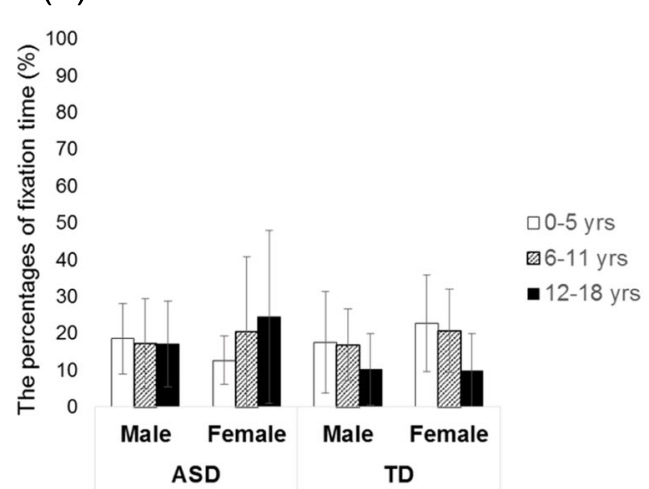

(d) TD
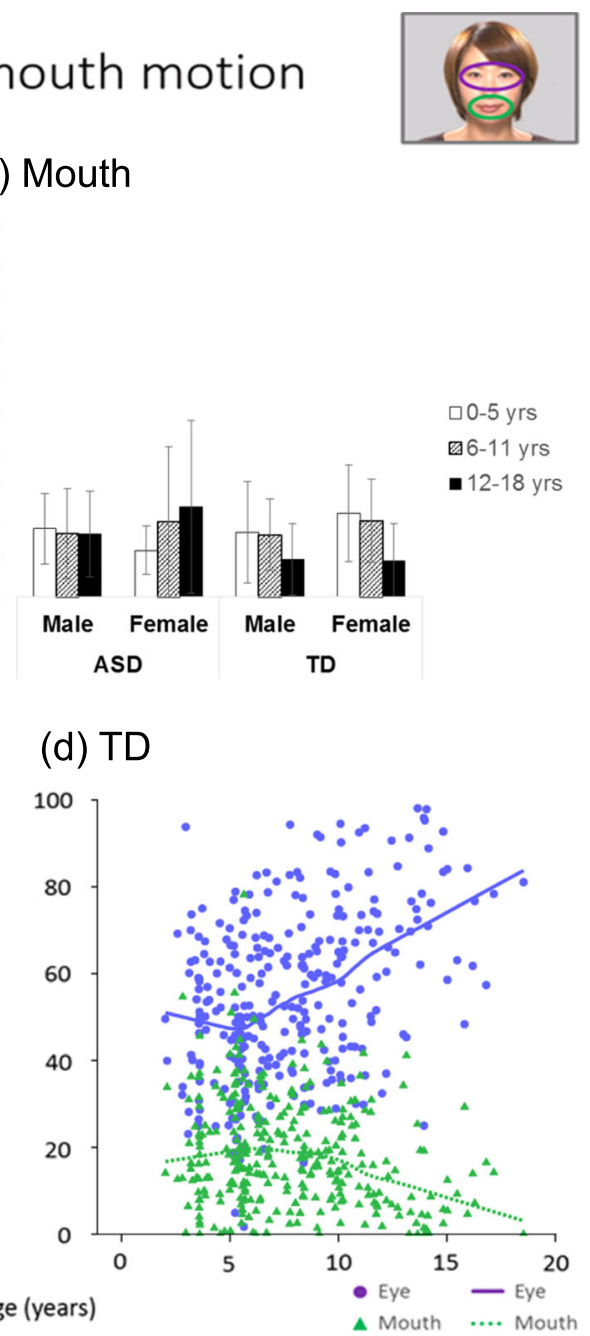

Fig. 2 Bar graphs and LOESS curves for "face without mouth motion." a, b The bar graphs of the ASD group and TD group for the percentage fixation times of the eye and mouth regions, respectively. Error bars indicate standard errors of the mean. c, d Scatter plots and LOESS curves of the ASD group and TD group, respectively. Purple circles and lines show data from the eye region, and yellow green triangles and dashed lines show data from the mouth region 


\section{Face with mouth motion}

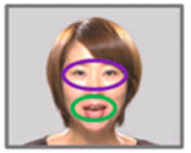

(a) Eye

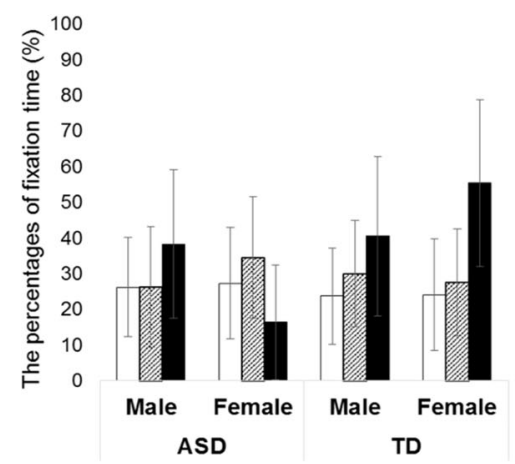

(c) ASD Male

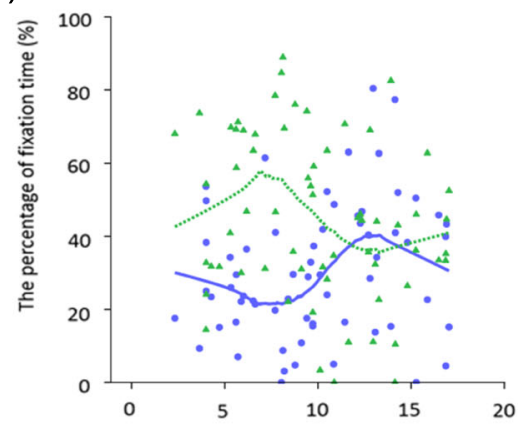

(e) ASD Female

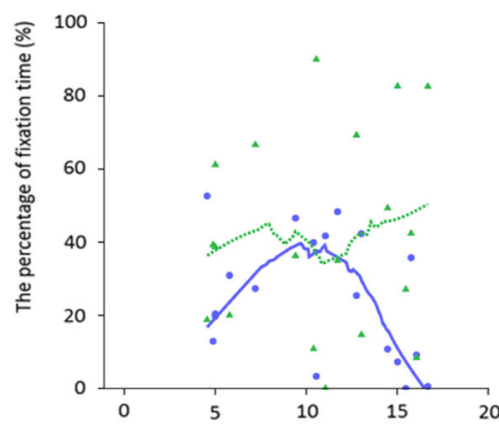

(b) Mouth

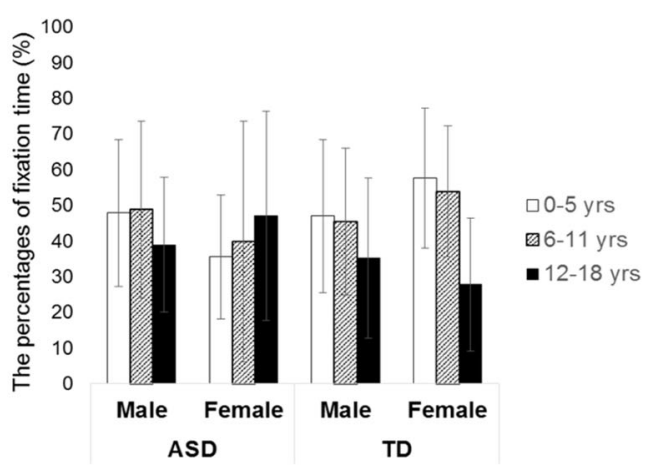

(d) TD Male

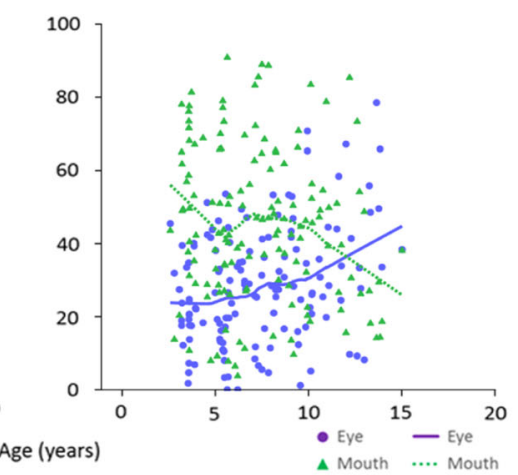

(f) TD Female

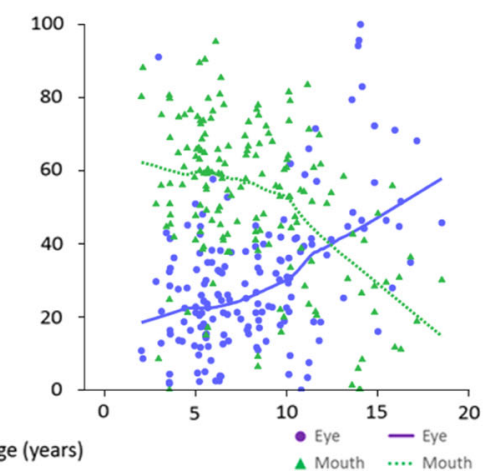

Fig. 3 Bar graphs and LOESS curves for "face with mouth motion." $\mathbf{a}, \mathbf{b}$ The bar graphs of the ASD group and TD group for the percentage fixation times of the eye and mouth regions, respectively. Error bars indicate standard errors of the mean. c-f Scatter plots and LOESS curves of the ASD group and TD group, respectively. Purple circles and lines show data from the eye region, and yellow green triangles and dashed lines show data from the mouth region

shows that there was a rise in attention to the mouth region in the ASD group after around 10 years of age, whereas there was a constant decline in attention to the mouth region in the TD group.

These results are shown in Table 2 and Fig. 3.

\section{Biological motion}

There were no main effects and interactions both in upright and inverted biological motion ( $\mathrm{Fs} \leqq 7.51, \mathrm{ps} \geqq .006$ ) (Table 2 and Fig. 4). Both the ASD and TD groups did not show a sharp rise or decline as shown in the LOESS plot in Fig. 4. 


\section{Biological Motion}

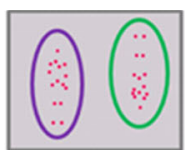

(a) Upright

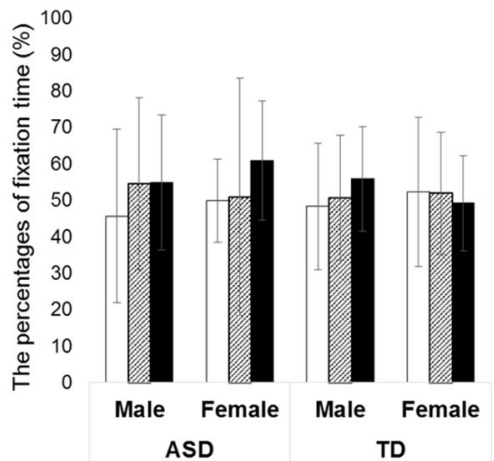

(c) ASD

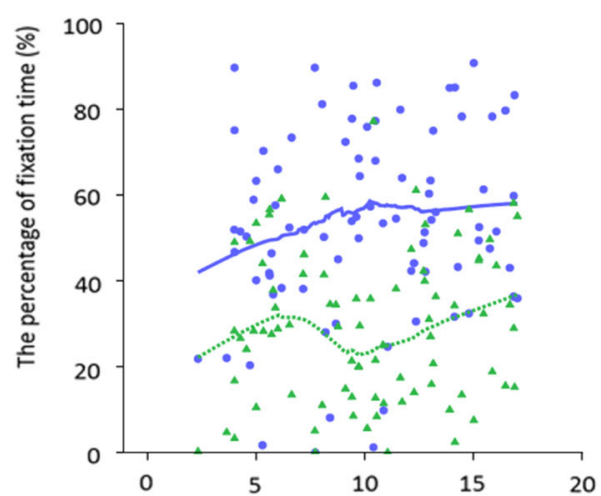

(b) Inverted

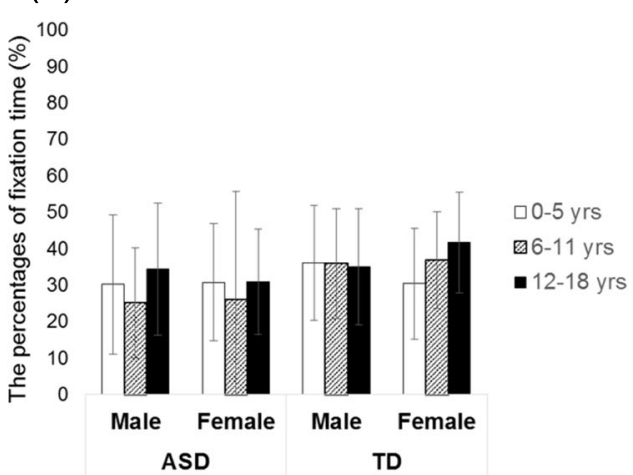

(d) TD

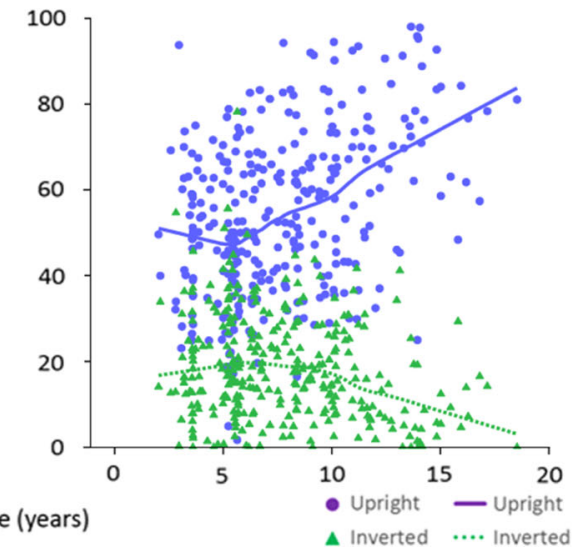

Fig. 4 Bar graphs and LOESS curves for "biological motion." a, b The bar graphs of the ASD group and TD group for the percentage fixation times of upright and inverted biological motion, respectively. Error bars indicate standard errors of the mean. $\mathbf{c}$, $\mathbf{d}$ Scatter plots and LOESS curves of the ASD group and TD group, respectively. Purple circles and lines show data from upright biological motion, and yellow green triangles and dashed lines show data from inverted biological motion

\section{The preference paradigm}

For the people region, the main effect of diagnosis was also significant $\left(F(1,378)=8.36, p=.004, \eta^{2}=0.022\right)$, and the TD group gazed longer at the people region than did the ASD group. In addition, there was a significant main effect of sex $\left(F(1,378)=10.39, p=.001, \eta^{2}=\right.$ 0.027), and the female group gazed longer at the people region than did the male group. The LOESS plot indicated that the percentage of fixation to the people region gradually decreased in the TD group and stabilized after around 5 years of age. The regression line for the ASD group declined until 10 years of age and showed a limited rise after this age.

The main effect of sex was also significant for the geometry region $\left(F(1,378)=10.59, p=.001, \eta^{2}=0.27\right)$, and the male group gazed longer at the geometry region than did the female group. Furthermore, the main effect of age was significant $\left(F(1,378)=6.09, p=.003, \eta^{2}=\right.$ 0.31 ). Post hoc comparisons showed that the $0-5$-year group gazed less at the geometry region than did the other two age groups (ps <.001). The regression lines of both the ASD and TD groups showed the opposite trend to that for the people region.

These results are shown in Table 2 and Fig. 5.

\section{Finger-pointing}

For the social region, there were no significant main effects or interactions ( $\mathrm{Fs} \leq 3.09$, ps $\geq .080$ ). For the geometry region, the main effect of diagnosis was significant $\left(F(1,378)=14.96, p<.001, \eta^{2}=0.038\right)$, and the TD group gazed longer at the geometry region than did the ASD group. Both the ASD and TD groups did not show 


\section{The preference paradigm}

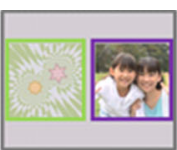

(a) People

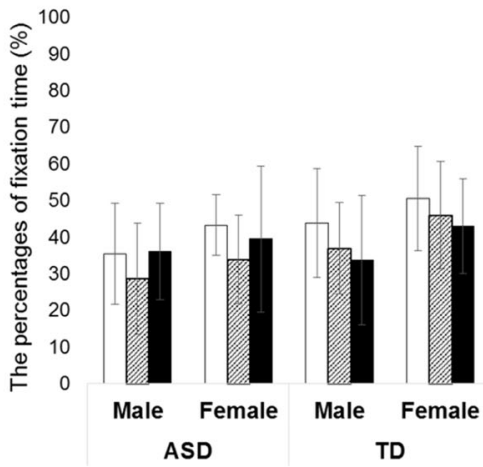

(c) ASD

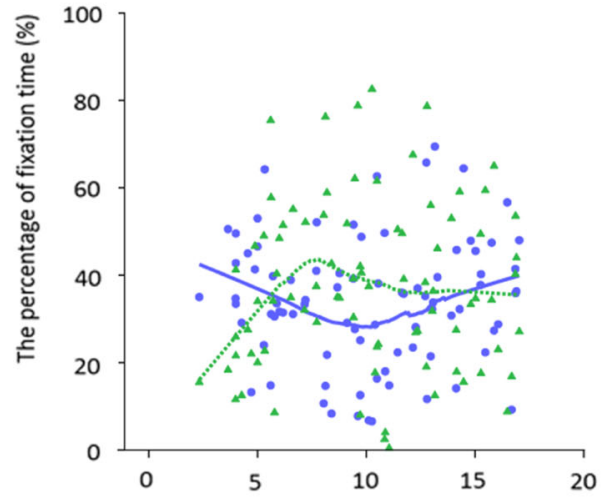

(b) Geometry

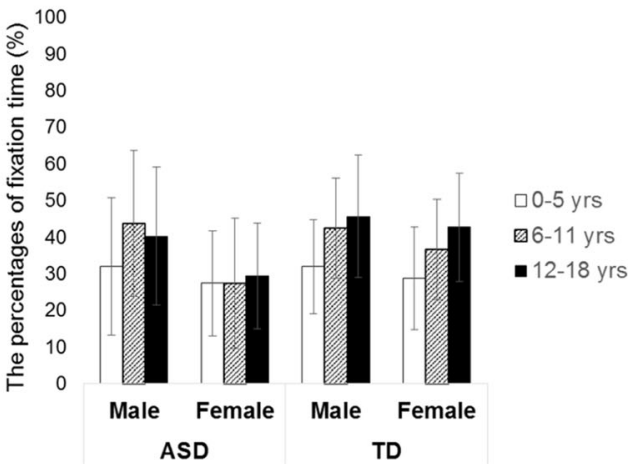

(d) TD

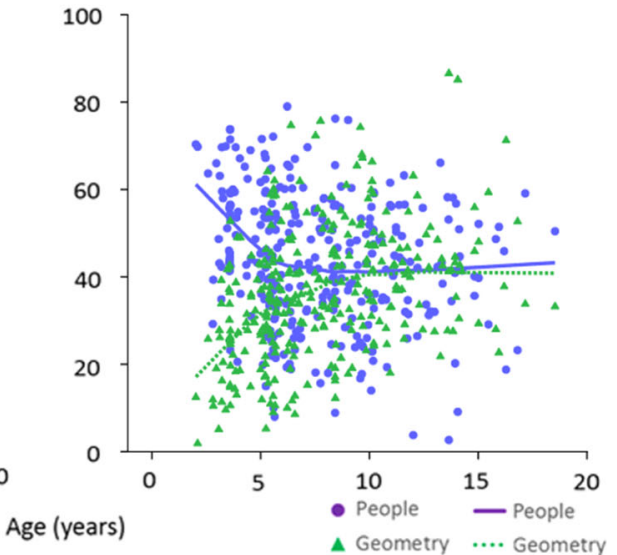

Fig. 5 Bar graphs and LOESS curves for the preference paradigm. $\mathbf{a}, \mathbf{b}$ The bar graphs of the ASD group and TD group for the percentage fixation times of the people and geometry regions, respectively. Error bars indicate standard errors of the mean. $\mathbf{c}$, $\mathbf{d}$ Scatter plots and LOESS curves of the ASD group and TD group, respectively. Purple circles and lines show data from the people region, and yellow green triangles and dashed lines show data from the geometry region

a sharp rise or decline in the LOESS plot. These results are shown in Table 2 and Fig. 6.

\section{Correlation analysis}

As there were main effects and interactions of the age group and sex in the face stimuli and the preference paradigm, we carried out partial correlation analysis using age and sex as covariates. At first, we carried out a correlation analysis between the SRS- 2 scores and the percentage fixation time to AoIs using age and sex as covariates. In the whole group analysis, we found that the SRS-2 total score was significantly negatively correlated with three items: inverted biological motion in biological motion in the $0-18$-year group and the people region in the preference paradigm in both the 0-18-year and 611-year groups. In addition, the percentage of fixation to the geometry region in finger-pointing showed a significant positive correlation with the SRS-2 total score in the ASD 0-18-year group. The other correlation coefficients were not significant, and all results are shown in Table 3. Next, we carried out a correlation analysis between the IQ/DQ scores and the percentage fixation time to AoIs in the ASD group using age and sex as covariates. There were no significant correlations, and all results are shown in Table 4.

\section{Discussion}

The aim of this study was to identify the developmental changes in gazing at social information in individuals with ASD and TD individuals from childhood to adolescence. In the face stimuli, the ANOVA results showed that the ratio of gazing at the eye region rose with 


\section{Pointing}

(a) Social area

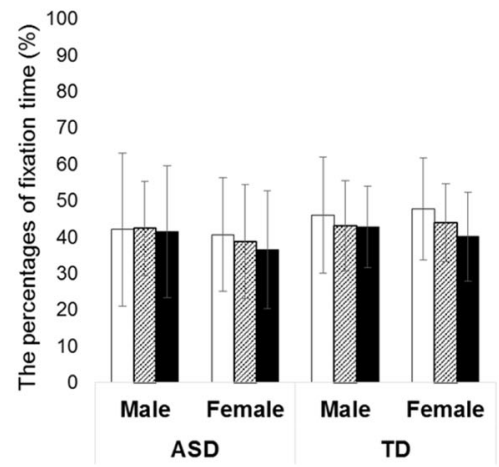

(c) ASD

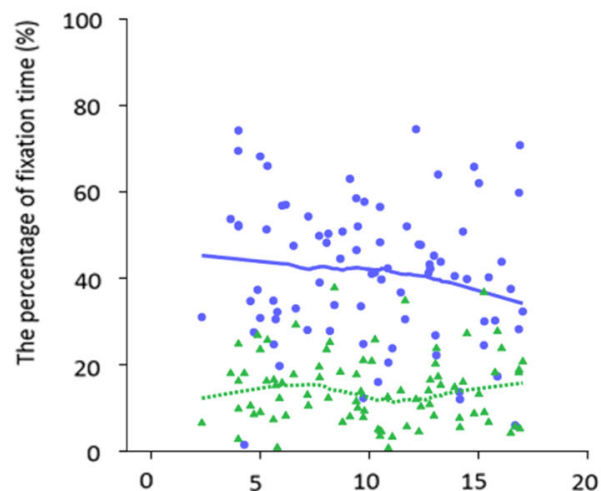

(b) Geometry

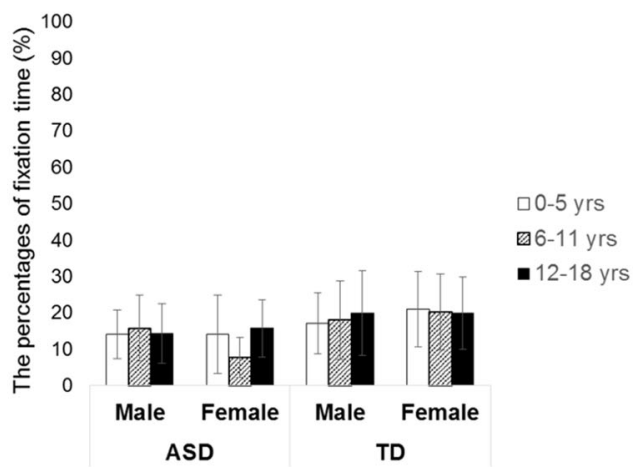

(d) TD
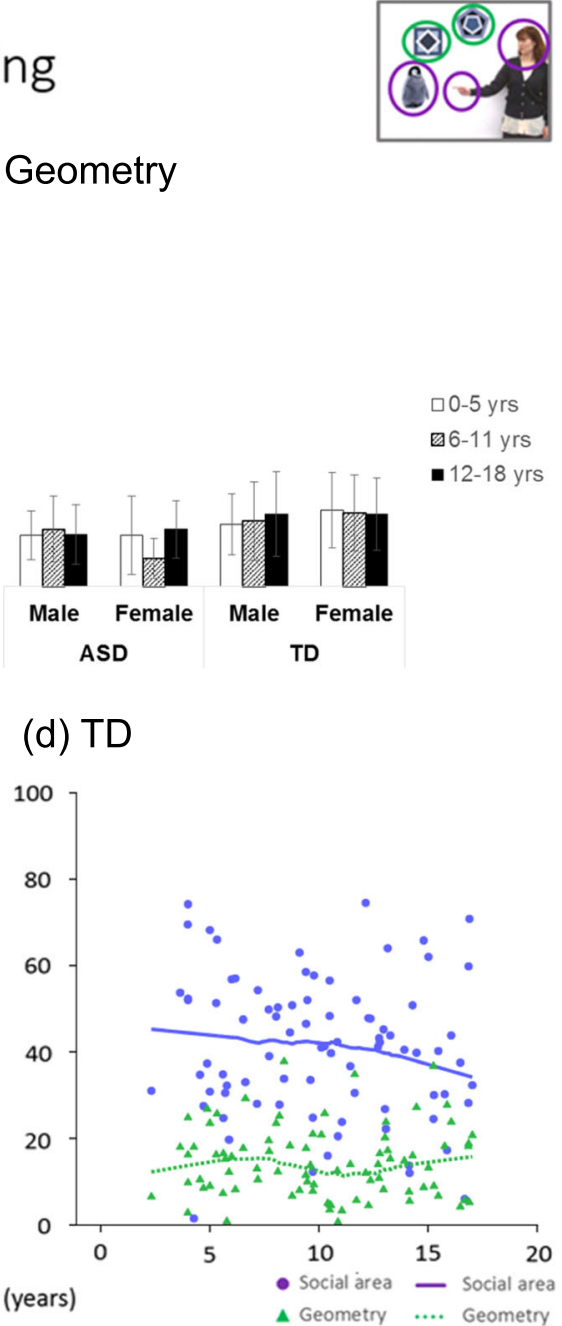

Fig. 6 Bar graphs and LOESS curves for "finger pointing." a, b The bar graphs of the ASD group and TD group for the percentage fixation times of objects with or without finger pointing, respectively. Error bars indicate standard errors of the mean. $\mathbf{c}$, $\mathbf{d}$ Scatter plots and LOESS curves of the ASD group and TD group, respectively. Purple circles and lines show data from objects with finger-pointing, and yellow green triangles and dashed lines show data from objects without finger-pointing

increasing age in the TD group. When being shown videos of faces "without mouth motion," the TD group showed reductions in the LOESS curves for the ratio of gazing at the eye region, along with increasing age until around 5 years of age and later showed a tendency for the curves to rise with increasing age. Conversely, in the ASD group, the LOESS curves of the ratio of gazing at the eye region continued to rise until around age 10 years and showed a tendency to decrease thereafter; a pattern especially pronounced in girls with ASD. The main effect of diagnosis was significant in the gaze ratio at the people region in the preference paradigm, with the ASD group's gaze ratio being lower than that of the TD group's.

In the ASD group, the ANOVA results for "face with mouth motion" did not show any increase in the gaze ratio at the eye region in line with increasing age as seen in the TD group. Especially, in female participants with ASD in "face with mouth motion," the LOESS regression curve in both face stimuli showed reduction in gazing at the eye region and increase in gazing at the mouth region at around age 10 years, although it is necessary to emphasize that ANOVA does not suffice to examine significant developmental changes. While the previous study that attempted to clarify the developmental changes in attention to social information in ASD targeted early childhood [37], we evaluated developmental changes in children throughout childhood and adolescence and delineated the developmental trajectories of complex behaviors. Our findings could be instrumental in elucidating the developmental and clinical characteristics of ASD. Another previous study reported on the 
Table 3 Results of partial correlation analysis between SRS-2 scores and the percentage fixation time to Aols using age and sex as covariates

\begin{tabular}{|c|c|c|c|}
\hline 0-18 years & $0-5$ years & 6-11 years & $12-18$ years \\
\hline$p$ & $p$ & $r$ & $r$ \\
\hline
\end{tabular}

\begin{tabular}{|c|c|c|c|c|c|c|c|c|c|}
\hline \multicolumn{10}{|l|}{ Whole group } \\
\hline \multirow[t]{2}{*}{ Face without mouth motion } & Eye & -.114 & .024 & .004 & .961 & -.044 & .555 & -.270 & .030 \\
\hline & Mouth & .095 & .062 & -.059 & .494 & .033 & .656 & .328 & .008 \\
\hline \multirow[t]{2}{*}{ Face without mouth motion } & Eye & -.127 & .012 & .007 & .940 & -.079 & .287 & -.261 & .036 \\
\hline & Mouth & .033 & .514 & -.014 & .870 & .016 & .833 & .127 & .314 \\
\hline \multirow[t]{2}{*}{ Biological motion } & Upright & .048 & .347 & -.061 & .477 & .069 & .354 & .094 & .456 \\
\hline & Inverted & -.150 & $.003^{*}$ & -.007 & .937 & -.212 & $.004 *$ & -.147 & .243 \\
\hline \multirow[t]{2}{*}{ The preference paradigm } & People & -.114 & .025 & -.107 & .215 & -.235 & $.001 *$ & .018 & .889 \\
\hline & Geometry & -.101 & .048 & -.065 & .451 & .021 & .779 & -.284 & .022 \\
\hline \multirow[t]{2}{*}{ Finger-pointing } & Social & -.025 & .620 & -.055 & .522 & -.040 & .593 & -.018 & .885 \\
\hline & Geometry & -.080 & .115 & -.074 & .392 & -.046 & .541 & -.190 & .129 \\
\hline \multicolumn{10}{|l|}{ ASD group } \\
\hline \multirow[t]{2}{*}{ Face without mouth motion } & Eye & -.082 & .464 & -.028 & .916 & .019 & .918 & -.236 & .227 \\
\hline & Mouth & .165 & .141 & .319 & .213 & .020 & .912 & .240 & .218 \\
\hline \multirow[t]{2}{*}{ Face without mouth motion } & Eye & -.114 & .310 & -.097 & .710 & -.112 & .540 & -.084 & .671 \\
\hline & Mouth & -.012 & .916 & .340 & .182 & -.181 & .323 & -.141 & .475 \\
\hline \multirow[t]{2}{*}{ Biological motion } & Upright & .072 & .525 & .183 & .482 & -.012 & .948 & .146 & .459 \\
\hline & Inverted & -.057 & .616 & -.212 & .415 & .129 & .483 & -.241 & .217 \\
\hline \multirow[t]{2}{*}{ The preference paradigm } & People & -.051 & .648 & .403 & .108 & -.226 & .213 & .010 & .960 \\
\hline & Geometry & .051 & .651 & .047 & .858 & .126 & .491 & -.186 & .343 \\
\hline \multirow[t]{2}{*}{ Finger-pointing } & Social & .035 & .755 & .258 & .316 & -.177 & .333 & .041 & .837 \\
\hline & Geometry & .313 & $.004 *$ & .602 & .011 & .357 & .045 & .127 & .518 \\
\hline \multicolumn{10}{|l|}{ TD group } \\
\hline \multirow[t]{2}{*}{ Face without mouth motion } & Eye & .022 & .705 & -.020 & .828 & .046 & .583 & .169 & .331 \\
\hline & Mouth & -.028 & .630 & -.035 & .704 & .010 & .904 & -.113 & .520 \\
\hline \multirow[t]{2}{*}{ Face without mouth motion } & Eye & -.007 & .898 & -.068 & .467 & -.025 & .763 & .124 & .479 \\
\hline & Mouth & .018 & .758 & .076 & .415 & .076 & .361 & -.200 & .249 \\
\hline \multirow[t]{2}{*}{ Biological motion } & Upright & -.029 & .616 & -.095 & .304 & .091 & .269 & -.271 & .115 \\
\hline & Inverted & .045 & .438 & .161 & .081 & -.083 & .318 & .272 & .115 \\
\hline \multirow[t]{2}{*}{ The preference paradigm } & People & .000 & .995 & -.020 & .829 & -.016 & .851 & .126 & .472 \\
\hline & Geometry & -.037 & .517 & -.068 & .462 & .046 & .581 & -.212 & .221 \\
\hline \multirow[t]{2}{*}{ Finger-pointing } & Social & .030 & .598 & -.037 & .689 & .060 & .466 & .192 & .270 \\
\hline & Geometry & .037 & .525 & .003 & .978 & .102 & .218 & -.132 & .450 \\
\hline
\end{tabular}

${ }^{*} p<.005$

developmental trajectories of social interaction from diagnosis through age 14 years, and these trajectories appeared to decline at ages greater than 10 years [48]. This clinical picture may be related to the reduction in gazing at the eye region and increase in gazing at the mouth region at around age 10 years that was observed in this study, and there may be qualitative differences in abnormal eye contact in ASD between individuals in early childhood and those older than 10 years.
Why, then, does the ratio of gazing at the eye region decrease in individuals with ASD older than 10 years as shown in the LOESS regression curve in both face stimuli? Some hypotheses are available to explain the lower attention to social information in individuals with ASD: the social motivation hypothesis [49], social aversion hypothesis [14, 50, 51], and "dynamics of a clinical phenotype" hypothesis [52, 53]. Social motivation refers to the mechanism by which people turn their attention 
Table 4 Results of partial correlation analysis between IQ/DQ scores and the percentage fixation time to Aols using age and sex as covariates in ASD group

\begin{tabular}{|c|c|c|c|c|c|c|c|c|c|}
\hline \multirow[t]{2}{*}{ ASD group } & & \multicolumn{2}{|c|}{$0-18$ years } & \multicolumn{2}{|c|}{$0-5$ years } & \multicolumn{2}{|c|}{$6-11$ years } & \multicolumn{2}{|c|}{$12-18$ years } \\
\hline & & $r$ & $p$ & $r$ & $p$ & $r$ & $p$ & $r$ & $p$ \\
\hline \multirow[t]{2}{*}{ Face without mouth motion } & Eye & -.008 & .941 & -.096 & .715 & -.061 & .742 & .133 & .501 \\
\hline & Mouth & .060 & .596 & .135 & .605 & .119 & .515 & -.076 & .700 \\
\hline \multirow[t]{2}{*}{ Face without mouth motion } & Eye & -.006 & .954 & -.317 & .215 & -.023 & .899 & .127 & .518 \\
\hline & Mouth & -.065 & .565 & .028 & .915 & -.083 & .652 & -.021 & .915 \\
\hline \multirow[t]{2}{*}{ Biological motion } & Upright & .001 & .991 & -.101 & .701 & -.042 & .818 & .161 & .414 \\
\hline & Inverted & .059 & .602 & -.077 & .768 & .288 & .109 & -.161 & .413 \\
\hline \multirow[t]{2}{*}{ The preference paradigm } & People & .037 & .742 & -.204 & .431 & -.056 & .761 & .268 & .169 \\
\hline & Geometry & -.071 & .526 & -.180 & .490 & .145 & .429 & -.338 & .079 \\
\hline \multirow[t]{2}{*}{ Finger-pointing } & Social & -.154 & .169 & .036 & .892 & -.152 & .407 & -.278 & .151 \\
\hline & Geometry & .120 & .284 & -.007 & .980 & .363 & .041 & -.089 & .652 \\
\hline
\end{tabular}

${ }^{*} p<.005$

to social stimuli, seek pleasure in social interaction and try to perceive it, and foster and maintain social connections [49]. According to the social motivation hypothesis, people with ASD have characteristically low social motivation, and because of this, their focus on social information is weak. In fact, the orbitofrontal-striatalamygdala circuit, which responds to social stimuli such as faces and social approval, has been repeatedly highlighted as abnormal in ASD [49]. The social aversion hypothesis contests that individuals with autism avoid gazing at social information as a result of the considerable resources required to process such social information, especially that of the eye region in people's faces. One related factor is likely to be anxiety, which is reported to reduce the gaze ratio at the eye region [54, 55]. Reportedly, the amygdala, which is a rapid detector of aversive environmental stimuli and gives rise to affective or behavioral states to allow for adaptive responses to potential threats [56], becomes strongly activated as a result of gazing at the eye region in individuals with anxiety disorder [57] and individuals with ASD $[51,58]$. This is probably why such individuals avoid looking other people in the eyes. Anxiety disorder is highly comorbid with ASD [59-61], with the onset of social anxiety disorders, in particular, reported to peak between the ages of 10 and 15 years [62]. Therefore, individuals with ASD after the age of 10 years showed an aversion to the eye region, which is typically processed as a reward in TD individuals [63], due to high anxiety. The "dynamics of a clinical phenotype" hypothesis states: "early emerging behavioral symptoms alter the child's self-directed patterns of attention, changing their experience of the environment and further restricting social learning opportunities" [52]. Thus, it is claimed that social behaviors including eye contact modulate cortical activation even in infants and that social experience may affect social behavior itself $[52,53]$. Because this was a cross-sectional study, we cannot deny the possibility that the older participants with ASD may have acquired the gaze behaviors described above through previous experience. The present findings do not provide evidence for any of these hypotheses; therefore, it is necessary to confirm them in the future.

Main-effects of diagnosis were shown for the people region in the preference paradigm and for the geometry region in the finger-pointing movies. Interestingly, although the people (social) region and the geometry region were set as AoIs in both stimuli, the participants in the ASD group gazed more briefly at the people region in the preference paradigm and the geometry region in the finger-pointing movie. These ambivalent results are considered to depend on the quality of the stimuli. Both the people and geometry regions moved in the preference paradigm, and only the social region moved in the finger-pointing movie. A previous study reported that individuals with ASD very attentively watched the hands that moved in the finger-pointing video [7]. Therefore, the possibility can also be considered that because the subjects were gazing attentively at the moving hands, there were no differences between the ASD and TD groups. Even if a researcher determines similar outcomes, opposite results may be obtained depending on the quality of the stimuli. Gazing behavior is very delicate, and researchers should be able to use the same stimuli across studies to enable comparisons.

For the preference paradigm, the ASD group reportedly gazed more briefly at the people region than did the TD group in previous studies targeting both children [26-30] and adults [22]. All the aforementioned studies with children [26-30] targeted early childhood, and to the best of our knowledge, our study was the first study to report on children during the course of childhood 
and adolescence. Considering our results, we contest that, regardless of age, the ratio of gazing at the people region in the preference paradigm may reflect the characteristic of sociability in ASD.

Regarding geometric shapes, the main effect of age group was significant. Although this was seen especially conspicuously in TD individuals, the participants gazed at geometric shapes longer as they grew older. This may imply that, along with increased age, individuals become more able to "search" for stimuli other than social stimuli. In other words, although individuals appear to look at social stimuli automatically [64-66], they may become able to divert their attention to non-social stimuli with increasing age.

Regarding the perception of biological motion under passive view conditions targeting children, the findings of previous studies are inconsistent, with some stating that children with ASD gaze less at biological motion [31-33], while others contesting that they gaze more [37]. Studies targeting adults found no significant difference between ASD and TD groups [22]. Studies conducted by Fujisawa et al. [37] and Fujioka et al. [22] found that individuals with ASD gazed at biological motion approximately the same as, or more than, TD individuals, using Gazefinder, as was used in our study [22, 37]. Therefore, the quality of the stimuli may also be related. A previous study reported that, if a nonsocial sound is attached to biological motion (response to nonsocial-physical contingencies that are disregarded by control children), or, in other words, if a clapping sound is simultaneously presented with the biological motion of hand clapping, children with ASD tend to look at the biological motion very attentively [31]. Biological motion in Gazefinder shows people dancing to a song, indicating a high rate of synchronization between sound and movement. Differentiation between ASD and TD may therefore be difficult with stimulation by Gazefinder.

For the partial correlation analysis using age and sex as covariates, there were a few significant correlations between the SRS- 2 total score and the percentage fixation time to AoIs. Previous research with adults with ASD using Gazefinder also reported this trend and concluded that the SRS-2 can measure a wide range of social deficits, whereas Gazefinder can only measure abnormality in eye contact, which is only one social deficit component [22]. However, some studies have reported significant correlations between attention to social information and the SRS-2 score [16] and other studies have reported significant correlations between attention to social information and the scores on other instruments, such as the Autism Diagnostic Observation Schedule (ADOS) [15, 17], while other studies have shown no significant correlations between them $[16,23]$. It will be necessary to clarify the types of abilities that are associated with attention to social information in the future, although it may be affected by the quality of the stimuli and the age of the participants. Furthermore, there were no significant correlations between the IQ/ DQ scores and the percentage fixation time to AoIs in the ASD group. Based on these results, attention to social information and IQ/DQ scores may be independent, at least in individuals with ASD.

This study made an important discovery regarding developmental changes in gazing at social information in the TD group. In TD group for "a face without mouth motion," the ANOVA and LOESS results showed that the gaze ratio at the eye region decreased and the ratio at the mouth region increased up to around 5 years of age, and afterward, the opposite trends were obtained. Considering previous findings that TD children gradually gaze more briefly at strong social information along with increasing age $[37,39,67]$ and that TD adults tended to gaze longer at the eye region than did TD children [11, 18], there is a discrepancy in that gazing at areas carrying strong social information decreases or does not change along with increasing age in childhood, whereas adults often gaze longer at such information than do children. The results of our study regarding faces without mouth motion explain this discrepancy. Then, why does the gaze ratio at the eye region decrease and the gaze ratio at the mouth region increase up to approximately 5 years of age in faces without mouth motion? A previous study reported that the fixation time to the mouth region at 0.5 years $(6$ months) old predicted expressive language at 2 years old [68], and participants up to 5 years who show significant language development may gaze longer at the mouth region at approximately 5 years old even in the faces without mouth motion. Subsequently, after 5 years of age, TD individuals could be able to divert their attention from the mouth and pay more attention to the eye region. Besides, in "face with mouth motion," the moving mouth was regarded to have attracted the attention of young children, and, as a result, gazing at the eye region showed an increase only in conjunction with age in this study. As such unique developmental changes in attention to social information cannot be confirmed with other stimuli, the eye region may hold a special place among the social information-carrying stimuli.

\section{Limitations}

We cannot deny the possibility that, because this was a cross-sectional study, the degree of severity and the degree of social interest might have differed according to the subjects' age group. In addition, in the ANOVA using age as a dependent variable for age group, there were group differences for age, sex, and/or diagnosis, in other words, there were differences in age among the 
sub-groups. These sub-group differences in age do not affect the interpretation of LOESS; however, they may have affected the ANOVA results when using the percentage fixation time to AoIs as the dependent variable. We believe that we will be able to further validate the findings of this study by clarifying the developmental changes in gazing at social information with longitudinal studies strictly controlling for the severity of ASD and age. In addition, especially in the ASD group, the sample size in each age group was somewhat small. On ANOVA analysis, the $p$ value of some items was below .05, which is a commonly used significance level, but not below .005 , which was the significance level we employed in this study adapted from Bonferroni corrections. Moreover, the sample size of the 0-5-year ASD group was considerably smaller than that of the other ASD age groups, as was the sample size of the 12-18-year TD group compared to the other TD age groups. As a result, it was challenging to obtain significant results with ANOVA, and the LOESS outcome may have been distorted. Although we revealed valuable findings in this study, it may be possible to reveal new trends by performing analysis with a larger and homogeneous group. In this study, we did not use gold-standard evaluations for diagnosis such as the ADOS and Autism Diagnostic Interview-Revised (ADI-R). As a previous study reported that the clinical diagnosis in the 2nd year of life without using the ADOS or ADI-R was stable [69] and the participants with ASD in this study were diagnosed by certified psychiatrists of the Japanese Board of Psychiatry or board-certified pediatricians of the Japan Pediatric Society, we are quite confident that the possibility of extreme misdiagnosis is very limited. However, from the viewpoint of group homogeneity and research, it would be preferable to evaluate participants using such tools in the future. In addition, a major limitation of this study is that no evaluation tools were used except for the SRS-2, and the IQ in the TD group was not measured. Therefore, the present results may have been affected by various factors. Future studies will be needed to measure and control for characteristics that affect attention to social information such as anxiety. All participants in this study were Japanese, and previous studies with Japanese and British adults/children have reported that Japanese participants looked longer in their eye area than did British participants [70, 71]. Confirmation is needed that our results can be replicated across ethnicities and cultures.

\section{Conclusions}

The purpose of this study was to identify, in children with ASD and TD children, the developmental changes in gazing at social information from childhood to adolescence. "Face without mouth motion" revealed characteristic developmental changes in the TD group, in which the gaze ratio at the eye region decreased up to approximately age 5 years but increased thereafter. The ASD group showed a reduction in gazing at the eye region around the age of 10 years. These results suggest that the eye region constitutes a special stimulus among the stimuli carrying social information. The main effect of diagnosis was significant in the gaze ratio at the people region in the preference paradigm, with the gaze ratio in the ASD group being lower than that in the TD group. The people region in the preference paradigm may be a stimulus that reflects the sociability of ASD individuals regardless of age. These developmental changes in the gaze ratio appear to be a major characteristic of ASD and were first clarified in our study that investigated developmental changes using a broader age group of 3 to 18 years. However, factors that could cause such developmental changes could not be identified in this study and warrant further research. Furthermore, if these factors are related to secondary disorders, as research progresses, it may be possible to predict secondary disorders and determine the methods and timing of intervention for these disorders by longitudinally measuring attention to social information.

\section{Abbreviations \\ ADI-R: Autism Diagnostic Interview-Revised; ADOS: Autism Diagnostic Observation Schedule; Aol: Area-of-interest; ANOVA: Analysis of variance; ASD: Autism spectrum disorder; DQ: Developmental quotient; IQ: Intelligent quotient; LOESS: Locally weighted scatterplot smoothing; SRS-2: Social Responsiveness Scale Second Edition; TD: Typically developed; WISC- IV: Wechsler Intelligence Scale for Children-Fourth Edition}

\section{Acknowledgements}

Not applicable.

\section{Authors' contributions}

TF conceived the study, recruited the participants, measured clinical data, performed the statistical analyses, interpreted clinical data, and wrote the manuscript. KJT and MS conceived the study, recruited the participants, diagnosed the participants, measured clinical data, and interpreted clinical data. MM, MK, and YMa conceived the study, recruited the participants, diagnosed the participants, and interpreted clinical data. YH conceived the study, recruited the participants, and interpreted clinical data. DC, SK, TY, YY, and SO measured clinical data. YMi, ST, HM, and AT recruited the participants, diagnosed the participants, and interpreted clinical data. KS and MN conceived the study. TK conceived the study and interpreted clinical data. HK conceived the study, recruited the participants, diagnosed the participants, interpreted clinical data, and provided critical review of the manuscript. All authors read and approved the final manuscript.

\section{Funding}

This study was partly funded by Grants-in-Aid for Scientific Research from the Japan Society for the Promotion of Science (16K17469), Research and Development of Supportive Device Technology for Medicine using ICT from Japan Agency for Medical Research and Development, and Research on Development of New Medical Devices from Japan Agency for Medical Research and Development.

\section{Availability of data and materials}

The datasets generated and/or analyzed during the current study are not publicly available because they were involved in a clinical trial but are available from the corresponding author on reasonable request. 


\section{Ethics approval and consent to participate}

The study protocol was approved by the ethics committee of each university and conformed to the tenets of the Declaration of Helsinki (as revised in 2000). After a complete explanation of the study, all participants or their parents/legal guardians provided written informed consent. JVC KENWOOD Corporation owns the rights to Gazefinder, samples of which can be found in figures 1-6. All persons appearing in Gazefinder have provided consent to appear in these images, and JVC KENWOOD Corporation has granted permission for use of these samples within this article.

\section{Consent for publication}

Not applicable.

\section{Competing interests}

The authors declare that they have no competing interests.

\section{Author details}

${ }^{1}$ Department of Science of Human Development, Humanities and Social Science, Faculty of Education, University of Fukui, Fukui, Fukui, Japan. ${ }^{2}$ Research Center for Child Mental Development, University of Fukui, Eiheiji, Fukui, Japan. ${ }^{3}$ Department of Child Development, United Graduate School of Child Development, Osaka University, Kanazawa University, Hamamatsu University School of Medicine, Chiba University, and University of Fukui, Suita, Osaka, Japan. ${ }^{4}$ Research Center for Child Mental Development, Hamamatsu University School of Medicine, Hamamatsu, Shizuoka, Japan. ${ }^{5}$ Department of Neuropsychiatry, Graduate School of Medicine, Hirosaki University, Hirosaki, Aomori, Japan. ${ }^{6}$ Research Center for Child Mental Development, Chiba University, Chiba, Chiba, Japan. ${ }^{7}$ Department of Pediatrics, Faculty of Medicine, Saga University, Saga, Saga, Japan. ${ }^{8}$ Research Center for Child Mental Development, Kanazawa University, Kanazawa, Ishikawa, Japan. ${ }^{9}$ Division of Child Neurology, Department of Brain and Neurosciences, Faculty of Medicine, Tottori University, Yonago, Tottori, Japan. ${ }^{10}$ Institute of Human and Social Sciences, Kanazawa University, Kanazawa, Ishikawa, Japan. ${ }^{11}$ Tottori University Hospital, Yonago, Tottori, Japan.

${ }^{12}$ Department of Child and Adolescent Psychological Medicine, University of Fukui Hospital, Eiheiji, Fukui, Japan. ${ }^{13}$ Development Center, Healthcare Business Division, JVCKENWOOD Corporation, Yokohama, Kanagawa, Japan. ${ }^{14}$ Department of Neuropsychiatry, Faculty of Medical Sciences, University of Fukui, Eiheiji, Fukui, Japan.

Received: 25 May 2019 Accepted: 7 February 2020

Published online: 09 April 2020

\section{References}

1. AmericanPsychiatricAssociation. Diagnostic and statistical manual of mental disorders: DSM-5. 5th ed: American Psychiatric Pub; 2013.

2. Seltzer MM, Krauss MW, Shattuck PT, Orsmond G, Swe A, Lord C. The symptoms of autism spectrum disorders in adolescence and adulthood. J Autism Dev Disord. 2003;33:565-81.

3. Vannucchi G, Masi G, Toni C, Dell'Osso L, Marazziti D, Perugi G. Clinical features, developmental course, and psychiatric comorbidity of adult autism spectrum disorders. CNS Spectr. 2014;19:157-64.

4. Chita-Tegmark M. Social attention in ASD: a review and meta-analysis of eye-tracking studies. Res Dev Disabil. 2016;48:79-93.

5. Chawarska K, Shic F. Looking but not seeing: atypical visual scanning and recognition of faces in 2 and 4-year-old children with autism spectrum disorder. J Autism Dev Disord. 2009;39:1663-72.

6. von Hofsten C, Uhlig H, Adell M, Kochukhova O. How children with autism look at events. J Autism Dev Disord. 2009;3:556-69.

7. Chawarska K, Macari S, Shic F. Context modulates attention to social scenes in toddlers with autism. J Child Psychol Psychiatry. 2012;53:903-13.

8. Chawarska K, Macari S, Shic F. Decreased spontaneous attention to social scenes in 6-month-old infants later diagnosed with autism spectrum disorders. Biol Psychiatry. 2013;74:195-203.

9. Saitovitch A, Bargiacchi A, Chabane N, Phillipe A, Brunelle F, Boddaert N, Samson Y, Zilbovicius M. Studying gaze abnormalities in autism: Which type of stimulus to use? Open J Psychiatry. 2013;03:32-8.

10. Shic F, Macari S, Chawarska K. Speech disturbs face scanning in 6-month-old infants who develop autism spectrum disorder. Biol Psychiatry. 2014;75:231-7.

11. Nakano $T$, Tanaka $K$, Endo $Y$, Yamane $Y$, Yamamoto $T$, Nakano $Y$, Ohta $H$, Kato N, Kitazawa S. Atypical gaze patterns in children and adults with autism spectrum disorders dissociated from developmental changes in gaze behaviour. Proc Biol Sci. 2010;277:2935-43.

12. Hosozawa M, Tanaka K, Shimizu T, Nakano T, Kitazawa S. How children with specific language impairment view social situations: an eye tracking study. Pediatrics. 2012;129:e1453-60.

13. Sasson N, Tsuchiya N, Hurley R, Couture SM, Penn DL, Adolphs R, Piven J. Orienting to social stimuli differentiates social cognitive impairment in autism and schizophrenia. Neuropsychologia. 2007;45:2580-8.

14. Dalton KM, Nacewicz BM, Johnstone T, Schaefer HS, Gernsbacher MA, Goldsmith HH, Alexander AL, Davidson RJ. Gaze fixation and the neural circuitry of face processing in autism. Nat Neurosci. 2005;8:519-26.

15. Jones W, Carr K, Klin A. Absence of preferential looking to the eyes of approaching adults predicts level of social disability in 2-year-old toddlers with autism spectrum disorder. Arch Gen Psychiatry. 2008;65:946-54.

16. Speer LL, Cook AE, McMahon WM, Clark E. Face processing in children with autism: effects of stimulus contents and type. Autism. 2007;11:265-77.

17. Shic F, Bradshaw J, Klin A, Scassellati B, Chawarska K. Limited activity monitoring in toddlers with autism spectrum disorder. Brain Res. 2011;1380: 246-54.

18. Amestoy A, Guillaud E, Bouvard MP, Cazalets JR. Developmental changes in face visual scanning in autism spectrum disorder as assessed by data-based analysis. Front Psychol. 2015;6.

19. Klin A, Jones W, Schultz R, Volkmar F, Cohen D. Visual fixation patterns during viewing of naturalistic social situations as predictors of social competence in individuals with autism. Arch Gen Psychiatry. 2002;59:809-16.

20. Rice K, Moriuchi JM, Jones W, Klin A. Parsing heterogeneity in autism spectrum disorders: visual scanning of dynamic social scenes in schoolaged children. J Am Acad Child Adolesc Psychiatry. 2012;51:238-48.

21. Pelphrey KA, Sasson NJ, Reznick JS, Paul G, Goldman BD, Piven J. Visual scanning of faces in autism. J Autism Dev Disord. 2002;32:249-61.

22. Fujioka T, Inohara K, Okamoto Y, Masuya Y, Ishitobi M, Saito DN, Jung M, Arai S, Matsumura Y, Fujisawa TX, et al. Gazefinder as a clinical supplementary tool for discriminating between autism spectrum disorder and typical development in male adolescents and adults. Mol Autism. 2016;7.

23. Corden B, Chilvers R, Skuse D. Avoidance of emotionally arousing stimuli predicts social-perceptual impairment in Asperger's syndrome. Neuropsychologia. 2008;46:137-47.

24. Sterling L, Dawson G, Webb S, Murias M, Munson J, Panagiotides H, Aylward E. The role of face familiarity in eye tracking of faces by individuals with autism spectrum disorders. J Autism Dev Disord. 2008;38:1666-75.

25. Boraston ZL, Corden B, Miles LK, Skuse DH, Blakemore SJ. Brief report: perception of genuine and posed smiles by individuals with autism. J Autism Dev Disord. 2008;38:574-80.

26. Pierce $\mathrm{K}$, Conant D, Hazin R, Stoner R, Desmond J. Preference for geometric patterns early in life as a risk factor for autism. Arch Gen Psychiatry. 2011;68: 101-9.

27. Shi L, Zhou Y, Ou J, Gong J, Wang S, Cui X, Lyu H, Zhao J, Luo X. Different visual preference patterns in response to simple and complex dynamic social stimuli in preschool-aged children with autism spectrum disorders. PLoS One. 2015;10:e0122280.

28. Moore A, Wozniak M, Yousef A, Barnes CC, Cha D, Courchesne E, Pierce K. The geometric preference subtype in ASD: identifying a consistent, earlyemerging phenomenon through eye tracking. Mol Autism. 2018;9:19.

29. Pierce K, Marinero S, Hazin R, McKenna B, Barnes CC, Malige A. Eye tracking reveals abnormal visual preference for geometric images as an early biomarker of an autism spectrum disorder subtype associated with increased symptom severity. Biol Psychiatry. 2016;79:657-66.

30. Franchini M, Wood de Wilde H, Glaser B, Gentaz E, Eliez S, Schaer M. Brief report: a preference for biological motion predicts a reduction in symptom severity 1 year later in preschoolers with autism spectrum disorders. Front Psychiatry. 2016;7:143.

31. Klin A, Lin DJ, Gorrindo P, Ramsay G, Jones W. Two-year-olds with autism orient to non-social contingencies rather than biological motion. Nature. 2009;459:257-61.

32. Annaz D, Campbell R, Coleman M, Milne E, Swettenham J. Young children with autism spectrum disorder do not preferentially attend to biological motion. J Autism Dev Disord. 2012;42:401-8.

33. Falck-Ytter T, Rehnberg E, Bolte S. Lack of visual orienting to biological motion and audiovisual synchrony in 3-year-olds with autism. PLoS One. 2013;8:e68816. 
34. Blake R, Turner LM, Smoski MJ, Pozdol SL, Stone WL. Visual recognition of biological motion is impaired in children with autism. Psychol Sci. 2003;14 151-7.

35. Falck-Ytter T, Fernell E, Hedvall AL, von Hofsten C, Gillberg C. Gaze performance in children with autism spectrum disorder when observing communicative actions. J Autism Dev Disord. 2012:42:2236-45.

36. Higuchi T, Ishizaki Y, Noritake A, Yanagimoto Y, Kobayashi H, Nakamura K, Kaneko K. Spatiotemporal characteristics of gaze of children with autism spectrum disorders while looking at classroom scenes. PLoS One. 2017;12: e0175912.

37. Fujisawa TX, Tanaka S, Saito DN, Kosaka H, Tomoda A. Visual attention for social information and salivary oxytocin levels in preschool children with autism spectrum disorders: an eye-tracking study. Front Neurosci. 2014;8:295.

38. van der Geest JN, Kemner C, Camfferman G, Verbaten MN, van Engeland H. Looking at images with human figures: comparison between autistic and normal children. J Autism Dev Disord. 2002;32:69-75

39. Frank MC, Vul E, Saxe R. Measuring the development of social attention using free-viewing. Infancy. 2012;17:355-75.

40. Lange J, Lappe M. A model of biological motion perception from configural form cues. J Neurosci. 2006;26:2894-906.

41. Salley B, Colombo J. Conceptualizing social attention in developmental research. Soc Dev. 2016;25:687-703.

42. Constantino JN, Gruber, C. The Social Responsiveness Scale (2nd ed.) (SRS-2) [Manual]. Torrance. Western Psychological Services; 2012.

43. Stickley A, Tachibana Y, Hashimoto K, Haraguchi H, Miyake A, Morokuma S, Nitta H, Oda M, Ohya Y, Senju A, et al. Assessment of autistic traits in children aged 2 to 4(1/2) years with the preschool version of the Social Responsiveness Scale (SRS-P): findings from Japan. Autism Res. 2017;10:852-65.

44. Kamio Y, Inada N, Moriwaki A, Kuroda M, Koyama T, Tsujii H, Kawakubo Y, Kuwabara H, Tsuchiya KJ, Uno Y, Constantino JN. Quantitative autistic traits ascertained in a national survey of 22529 Japanese schoolchildren. Acta Psychiatr Scand. 2013;128:45-53.

45. Cleveland WS. Robust locally weighted regression and smoothing scatterplots. Alcohol Clin Exp Res. 1979;74:829-36.

46. Cleveland WS, Devlin SJ. Locally weighted regression: an approach to regression analysis by local fitting. J Am Stat Assoc. 1988;83:596.

47. Shen C, Brooks MJ, van den Hengel A. Fast global kernel density mode seeking: applications to localization and tracking. IEEE Trans Image Process. 2007;16:1457-69.

48. Fountain C, Winter AS, Bearman PS. Six developmental trajectories characterize children with autism. Pediatrics. 2012;129:e1112-20.

49. Chevallier C, Kohls G, Troiani V, Brodkin ES, Schultz RT. The social motivation theory of autism. Trends Cogn Sci. 2012;16:231-9.

50. Ishitobi M, Kosaka H, Omori M, Matsumura Y, Munesue T, Mizukami K, Shimoyama T, Murata T, Sadato N, Okazawa H, Wada Y. Differential amygdala response to lower face in patients with autistic spectrum disorders: an fMRI study. Res Autism Spect Dis. 2011;5:910-9.

51. Tottenham N, Hertzig ME, Gillespie-Lynch K, Gilhooly T, Millner AJ, Casey BJ. Elevated amygdala response to faces and gaze aversion in autism spectrum disorder. Soc Cogn Affect Neurosci. 2014;9:106-17.

52. Jones EJ, Gliga T, Bedford R, Charman T, Johnson MH. Developmental pathways to autism: a review of prospective studies of infants at risk. Neurosci Biobehav Rev. 2014:39:1-33.

53. Senju A, Johnson MH. The eye contact effect: mechanisms and development. Trends Cogn Sci. 2009;13:127-34.

54. Horley K, Williams LM, Gonsalvez C, Gordon E. Social phobics do not see eye to eye: a visual scanpath study of emotional expression processing. J Anxiety Disord. 2003;17:33-44.

55. Kleberg JL, Hogstrom J, Nord M, Bolte S, Serlachius E, Falck-Ytter T. Autistic traits and symptoms of social anxiety are differentially related to attention to others' eyes in social anxiety disorder. J Autism Dev Disord. 2017;47: 3814-21.

56. Janak PH, Tye KM. From circuits to behaviour in the amygdala. Nature. 2015; 517:284-92.

57. Phan KL, Fitzgerald DA, Nathan PJ, Tancer ME. Association between amygdala hyperactivity to harsh faces and severity of social anxiety in generalized social phobia. Biol Psychiatry. 2006;59:424-9.

58. Hadjikhani N, Asberg Johnels J, Zurcher NR, Lassalle A, Guillon Q, Hippolyte L, Billstedt E, Ward N, Lemonnier E, Gillberg C. Look me in the eyes: constraining gaze in the eye-region provokes abnormally high subcortical activation in autism. Sci Rep. 2017;7:3163.
59. Belardinelli C, Raza M. Comorbid behavioral problems and psychiatric disorders in autism spectrum disorders. J Childhood Dev Dis. 2016;02.

60. Buck TR, Viskochil J, Farley M, Coon H, McMahon WM, Morgan J, Bilder DA. Psychiatric comorbidity and medication use in adults with autism spectrum disorder. J Autism Dev Disord. 2014;44:3063-71.

61. Lugnegard T, Hallerback MU, Gillberg C. Psychiatric comorbidity in young adults with a clinical diagnosis of Asperger syndrome. Res Dev Disabil. 2011 32:1910-7.

62. Schneier FR, Johnson J, Hornig CD, Liebowitz MR, Weissman MM. Social phobia. Comorbidity and morbidity in an epidemiologic sample. Arch Gen Psychiatry. 1992:49:282-8.

63. Hietanen JK. Affective eye contact: an integrative review. Front Psychol. 2018:9:1587.

64. Fletcher-Watson S, Leekam SR, Benson V, Frank MC, Findlay JM. Eyemovements reveal attention to social information in autism spectrum disorder. Neuropsychologia. 2009;47:248-57.

65. Birmingham E, Bischof WF, Kingstone A. Social attention and real-world scenes: the roles of action, competition and social content. Quart J Exp Psychol. 2008;61:986-98.

66. van der Geest JN, Kemner C, Verbaten MN, van Engeland H. Gaze behavior of children with pervasive developmental disorder toward human faces: a fixation time study. J Child Psychol Psychiatry. 2002:43:669-78.

67. Nishizato M, Fujisawa TX, Kosaka H, Tomoda A. Developmental changes in social attention and oxytocin levels in infants and children. Sci Rep. 2017;7: 2540.

68. Young GS, Merin N, Rogers SJ, Ozonoff S. Gaze behavior and affect at 6 months: predicting clinical outcomes and language development in typically developing infants and infants at risk for autism. Dev Sci. 2009;12: 798-814.

69. Charman T, Baron-Cohen S, Swettenham J, Baird G, Drew A, Cox A Predicting language outcome in infants with autism and pervasive developmental disorder. Int J Lang Commun Disord. 2003;38:265-85.

70. Senju A, Vernetti A, Kikuchi $Y$, Akechi H, Hasegawa T. Cultural modulation of face and gaze scanning in young children. PLoS One. 2013;8:e74017.

71. Senju A, Vernetti A, Kikuchi Y, Akechi H, Hasegawa T, Johnson MH. Cultural background modulates how we look at other persons' gaze. Int J Behav Dev. 2013;37:131-6.

\section{Publisher's Note}

Springer Nature remains neutral with regard to jurisdictional claims in published maps and institutional affiliations.

Ready to submit your research? Choose BMC and benefit from:

- fast, convenient online submission

- thorough peer review by experienced researchers in your field

- rapid publication on acceptance

- support for research data, including large and complex data types

- gold Open Access which fosters wider collaboration and increased citations

- maximum visibility for your research: over $100 \mathrm{M}$ website views per year

At $\mathrm{BMC}$, research is always in progress.

Learn more biomedcentral.com/submissions 